\title{
Children with cancer during COVID-19 pandemic. Early experience in Mexico.
}

\author{
Javier López-Aguilar ${ }^{1}$, Rocío Cárdenas-Navarrete ${ }^{1}$, Alfredo Simental Toba ${ }^{1}$, Gissel \\ Soto-Pérez ${ }^{1}$, Daniel Pacheco-Rosas ${ }^{1}$, Patricia Thomé-Ortiz ${ }^{1}$, Jorge Martín-Trejo ${ }^{1}$, \\ Guillermo Vázquez-Rosales ${ }^{1}$, and Guadalupe Miranda-Novales ${ }^{1}$ \\ ${ }^{1}$ IMSS
}

July 31, 2020

\begin{abstract}
Reports of children with cancer and COVID-19 from U.S.A and Europe had informed most patients presented with mild disease. Experience from upper and lower middle-income countries is needed to corroborate these findings. This report describes the experience with cancer patients in a tertiary care level hospital, in Mexico City. Of 24 symptomatic patients, 14 had a positive RT-PCR test. Eight required low-flow supplemental oxygen. None required critical care. One patient died due to pulmonary hemorrhage. Even though the infection seems to have an uncomplicated clinical course, it is necessary to ensure that all seeking medical attention do so safely.
\end{abstract}

\section{Abstract}

Reports of children with cancer and COVID-19 from U.S.A and Europe had informed most patients presented with mild disease. Experience from upper and lower middle-income countries is needed to corroborate these findings. This report describes the experience with cancer patients in a tertiary care level hospital, in Mexico City. Of 24 symptomatic patients, 14 had a positive RT-PCR test. Eight required low-flow supplemental oxygen. None required critical care. One patient died due to pulmonary hemorrhage. Even though the infection seems to have an uncomplicated clinical course, it is necessary to ensure that all seeking medical attention do so safely.

Introduction

In December 2019 in Wuhan, Province of Hubei, China, initiated the beginning of a devastating pandemic. The first cases of severe pneumonia brought a great deal of concern, later, a high lethality was seen in patients with comorbidities. However, cases in children showed a different course of the disease, with most having mild symptoms, transient fever, cough, and rarely acute respiratory failure that progressed to the need for care in an intensive care unit. In one of the first descriptions in China, Dong et al reported in a group of 2143 suspected or confirmed patients that the median affected age was 7 years, with the majority (89\%) of confirmed cases with mild or moderate symptoms. Seven of the 13 critical patients were under 1 year old. ${ }^{1}$

Recommendations were published for the attention and care of children and adolescents with cancer or leukemia during the pandemic, ${ }^{2}$ and an invitation and call for sharing experiences was requested by Eric Bouffet on March 2020. ${ }^{3}$ Several countries had been affected with more intensity. Reports of children with cancer from U.S.A and Italy had informed most of the affected patients had a mild disease and very few of them required critical care. ${ }^{4-7}$ 
The aim of this report is to share the experience with cancer patients cared for in a tertiary care level hospital, in Mexico City.

Results.

The study was conducted at the Pediatric Hospital, National Medical Center, XXI Century, of the Mexican Social Security Institute. All patients 17 years and younger who underwent SARS-CoV-2 testing were included from March 20, 2020 - June 20, 2020. Only symptomatic patients were tested, but it was not strictly necessary for them to comply with the criteria for COVID-19 illness, and fever could be the only sign. Data on demographics, clinical symptoms, haemato-oncological disease, complete blood count, inflammatory markers, imaging, and treatment were obtained from medical records. Management was established according to the hospitals' Clinical Guideline. Of a total of 226 symptomatic patients, 73 (32\%) tested positive for SARS-CoV-2 (real time RT-PCR). Of 24 children with cancer, during this period, 14 (58\%) had a positive result. Median age 10 years (range 3-15); male 8 and female 6. Source of contagion was a household member in two cases, three got infected at the referral hospital, and unknown for 9 children. Types of cancer: nine leukemias, two hepatoblastomas, one lymphoma, one osteosarcoma, and one central nervous system tumor (Table 1). All patients were hospitalized. Eight patients presented as an episode of fever and neutropenia (two with pneumonia and one with neutropenic colon), three patients were referred from other hospitals due to signs and symptoms of leukemia/lymphoma and RT-PCR test were taken at admittance. One patient was hospitalized due to cancer morbidity, and one with relapse of her leukemia. The last patient with leukemia had a complicated bacterial pneumonia, with an initial negative result for SARS-CoV-2, later he developed pachypleuritis and was referred for surgery, 16 days after his first test, he had a positive result. In all, 14/14 (100\%) had fever, 7/14 (50\%) cough, abdominal pain 3/14 (21\%), diarrhea 2/14 (14\%), and one dyspnea (7\%). Despite few respiratory symptoms, eight had oxygen saturation $<92 \%$ and required low-flow supplemental oxygen. None required critical care.

Laboratory findings: eight children had neutropenia $<500 / \mathrm{mm}^{3} ;$ lymphocytes median count 740 (range 2001610 excluding the three with a recent leukemia/lymphoma diagnosis), C-reactive protein median value 85 $\mathrm{mg} / \mathrm{L}$ (range 1.2-170), D-dimer median value $956 \mathrm{ng} / \mathrm{mL}$ (range 250-2779), and ferritin (only from 7 patients) median value $2587 \mathrm{ng} / \mathrm{mL}$ (range 583-3369). Bacterial cultures and other virologic test were negative. Radiology abnormalities were reported for 11 patients. A reticular interstitial pattern in four patients, defined consolidation in the lower inferior lobe in two, peripheral ground glass opacities in two, and nodular pleural thickening in one in lower inferior lobe. The patient with osteosarcoma had pulmonary metastases documented the previous month and loculated empyema in the patient with complicated pneumonia. In three the imaging studies were normal.

Standard treatment was used for all patients, patients with fever and neutropenia received piperacillin/tazobactam, in combination with vancomycin for the two patients with pneumonia, corticosteroids were employed according to the planned chemotherapy protocol, and filgrastim was indicated if neutrophil count $<1000 / \mathrm{mm}^{3}$ in selected patients. The patient with osteosarcoma and pulmonary metastases had sudden deterioration and died on the fifth day of hospitalization. The cause of death was pulmonary hemorrhage.

As at June 20, 2020, 13 patients had a negative test for SARS-CoV-2 in a median time of 27 days (range 14-56). Median hospital stay was 11 days (range 4 to 37). Cancer treatment was delayed until obtaining the negative result of the RT-PCR test.

Discussion

Although it has been reported that children are not the population most affected by the SARS-CoV-2 virus, information in special groups, such as cancer patients, require attention. The Leukemia Committee of the French Society for the fight against Cancers and Leukemias in children and adolescents, made a general recommendation for children with ALL and confirmed SARS-CoV-2 infection, to discuss the stopping and/or postponing of all chemotherapies. Decisions had to be analysed case by case. ${ }^{2}$ This recommendation coincides with that of Liang et al. adult patients, based on a greater severity of SARS-CoV-2 observed in cancer 
patients in China. ${ }^{8}$ However, these recommendations were made in the first months of the pandemic and now with the different reports around the world ${ }^{4-7,9}$ which informed a favourable evolution in most patients, the decision to postpone or stop treatment should be reconsidered. Unfortunately reports from countries like Mexico are scarce ${ }^{10}$ and it is difficult to predict that most cases of COVID-19 in children with cancer will recover with no complications.

In Mexico, at the beginning of the pandemic, only symptomatic patients were eligible for testing. The Pediatric Hospital at National Medical Center serves as a hybrid hospital, as all scheduled consultations and surgeries were suspended, the hospital continued to provide care to patients with various underlying conditions. Areas for COVID-19 patient care were adapted. Later, there was a need for testing not only for symptomatic patients, but for all patients seeking medical attention. Even though the infection with SARS-CoV-2 seems to have an uncomplicated clinical course in most of the patients, it is necessary to ensure that all of them who receive their chemotherapy scheme do so safely.

Conflict of interest

The authors certify that they have no affiliations with or involvement in any organization or entity with any financial interest, or non-financial interest (such as personal or professional relationships, affiliations, knowledge or beliefs) in the subject matter or materials discussed in this manuscript.

Acknowledgments

We thank all the health-care workers who have participated in the care of patients with COVID-19.

References

1. Dong Y, Mo X, Hu Y et al. Epidemiological characteristics of 2143 pediatric patients with 2019 coronavirus disease in China. Pediatrics . 2020; doi: 10.1542/peds.2020-0702

2. Baruchel A, Bertrand Y, Boisse, $\mathrm{N}$ et al. and SFCE Leukemia Committee. COVID-19 and acute lymphoblastic leukemias of children and adolescents: First recommendations of the Leukemia committee of the French Society for the fight against Cancers and Leukemias in children and adolescents (SFCE). Bulletin du cancer . 2020;107(6): 629-632. https://doi.org/10.1016/j.bulcan.2020.04.003

3. Bouffet E, Challinor J, Sullivan M, Biondi A, Rodriguez-Galindo C, Pritchard-Jones K. Early advice on managing children with cancer during the COVID-19 pandemic and a call for sharing experiences.Pediatr Blood Cancer . 2020:e28327.https://doi.org/10.1002/pbc.2832\%.

4. Boulad F, Kamboj M, Bouvier N, Mauguen A, Kung AL. COVID-19 in children with cancer in New York City [published online ahead of print, 2020 May 13]. JAMA Oncol . 2020;e202028. doi:10.1001/jamaoncol.2020.2028

5. Gampel B, Troullioud Lucas AG, Broglie L et al. COVID-19 disease in New York City pediatric hematology and oncology patients [published online ahead of print, 2020 Jun 26]. Pediatr Blood Cancer . 2020;e28420. doi:10.1002/pbc.28420

6. Ferrari A, Zecca M, Rizzari C, et al. Children with cancer in the time of COVID-19: An 8-week report from the six pediatric onco-hematology centers in Lombardia, Italy. Pediatr Blood Cancer 2020; 67: e28410. doi:10.1002/pbc.28410

7. Bisogno G, Provenzi M, Zama D et al. Clinical characteristics and outcome of SARS-CoV-2 infection in Italian pediatric oncology patients: a study from the Infectious Diseases Working Group of the AIEOP [published online ahead of print, 2020 Jul 11]. J Pediatric Infect Dis Soc . 2020;piaa088. doi:10.1093/jpids/piaa088

8. Liang W, Guan W, Chen R, et al. Cancer patients in SARS-CoV-2 infection: a nationwide analysis in China. Lancet Oncol . 2020;21(3):335-337. doi:10.1016/S1470-2045(20)30096-6

9. de Rojas T, Pérez-Martínez A, Cela E, et al. COVID-19 infection in children and adolescents with cancer in Madrid. Pediatr Blood Cancer . 2020;67(7):e28397. doi:10.1002/pbc.28397

10. Flores V, Miranda R, Merino L et al. SARS-CoV-2 infection in children with febrile neutropenia. Ann Hematol 99, 1941-1942 (2020). https://doi.org/10.1007/s00277-020-04115-1 


\section{Hosted file}

Table 1.docx available at https://authorea.com/users/347748/articles/473312-children-withcancer-during-covid-19-pandemic-early-experience-in-mexico 